10 years ESJ

Special edition

\title{
The Importance of Multilingual Information and Plain English in Response to the COVID-19 Pandemic
}

\author{
Barbara Cappuzzo \\ University of Palermo, Italy
}

Doi: 10.19044/esj.2021.v17n30p37

Submitted: 21 June 2021

Accepted: 15 July 2021

Published: 10 September 2021
Copyright 2021 Author(s)

Under Creative Commons BY-NC-ND

4.0 OPEN ACCESS

Cite As:

Cappuzzo B. (2021). The Importance of Multilingual Information and Plain English in Response to the COVID-19 Pandemic. European Scientific Journal, ESJ, 17 (30), 37.

https://doi.org/10.19044/esj.2021.v17n30p37

\section{Abstract}

Health is a common issue for all human beings. As a consequence, everyone in the world has in some way to cope with the language of medicine. This is true now more than ever due to the global health crisis caused by the current COVID-19 pandemic, which has introduced a great amount of terms, previously mostly used by epidemiologists and statisticians, but which now have entered the daily lexicon of many languages. As the medium of international scientific communication, English is the language of worldwide information about the pandemic, and the main source of terms and expressions for other languages. The impact of the COVID-19 pandemic on English lexicon has been so deep that the Oxford English Dictionary Online (OED) released special updates in 2020 to fulfil the need to document the phenomenon. However, previous studies (Khan et al. 2020; Deang and Salazar 2021) have highlighted the important question concerning the existence of several ethnic minorities who have Limited English Proficiency (LEP) and therefore do not receive sufficient and appropriate information to defend themselves adequately against SARS-CoV-2, the virus we have all been fighting for more than one year now. The aim of this study is to highlight the importance of language and translation as essential components to provide all demographic groups/communities with access to COVID-19-related information in languages other than English and enable them to follow official health key rules. The main websites of Italian governmental and nongovernmental institutions were investigated, and the analysis focused on the availability and type of content of the multilingual material, as well as on 
information accessibility and clarity. The results showed important differences in the number of available languages and, even more, in the level of intelligibility of COVID-19 material in the English language. In this respect, this study intends to foster the use of plain English in the dissemination material provided by the websites of the main healthcare public institutions in Italy, a country with an ever-increasing number of registered foreigners, the majority born in non-EU countries.

Keywords: Multilingual information, linguistic minorities, plain English

\section{Introduction}

This study is basically divided into two main parts. The first part explores the availability of COVID-19-related information as provided by some of the main governmental and non-governmental Italian websites. The second part focuses on the importance of using plain English in Italian public health written communication so as to allow linguistic minorities with limited English proficiency (LEP) to easily access information about COVID-19 and be able to follow the institutional key rules in force in Italy. The study will be preceded by a short overview of how English as the language of international communication and science has changed due to the current pandemic.

English and the COVID-19 pandemic

Language is the most immediate evidence of the changes that take place in our society. The COVID-19 pandemic has brought important changes in all spheres of life at a global level, and the world's languages have had to adapt to these changes by coining neologisms or using existing words and terms employed with new meanings or new shades of meaning. As the medium of international communication and science, English is the language that has had the greatest impact on other languages, especially those traditionally open to foreign words, such as, for instance, Italian. Most importantly, the lexical changes that English has undergone due to the COVID-19 pandemic have been so numerous that the Oxford English Dictionary Online (OED), typically updated on a quarterly basis, published various special releases in 2020 to document the phenomenon. Previously, the $O E D$ 's lexicographers usually selected a "Word of the Year", namely a word that they considered could embody the spirit of a given year on the basis of its influence on English. They could not select a single word last year because 2020 caused an unprecedented lexical change in the language, which led lexicographers to dedicate entire sections to the language of the COVID-19 pandemic. The OED's editors have documented several coronavirus-related linguistic shifts that have occurred in the English language, first and foremost the presence of technical scientific terms that before the pandemic were mostly used by epidemiologists and statisticians and that are now employed in news broadcasts and political 
speeches. Some significant examples are community transmission, herd immunity, mortality rate, reproduction number, all terms that are becoming increasingly widespread in everyday exchange too.

As the language of international communication and science, English has had a great influence on other languages, especially Italian, German, Dutch, Filipino, and Telugu (while other languages like Spanish, French, Portuguese, Chinese, Arabic, and Zulu have preferred equivalent terms). The term lockdown, for example, is extensively used in the Italian language at all levels of communication. This is because, when used in Italian, lockdown is unequivocal, since it is employed exclusively with reference to the COVID19 pandemic. Moreover, lockdown is concise and semantically all-inclusive as it encompasses the several connotations of meaning contained in its equivalents (for in-depth investigation of lockdown in the Italian language, see Cappuzzo 2020). There is also the interesting case of another Anglicism in Italian, i.e. droplet, which is used not only to refer to the small airborne drops of saliva and respiratory secretions by which the virus can be transmitted but also with the extended meaning of security distance that people have to maintain to prevent the spread of the virus. Moreover, due to further semantic extension, droplet is also being used to refer to the rule itself that imposes the security distance for the prevention of COVID-19 viral transmission (Treccani, www.treccani.it).

Deang and Salazar (2021) highlight that the prevalence of Anglicisms may be troubling, and that "citizens of countries like Italy and Japan have criticized their leaders for their over-reliance on English loanwords that they feel obscures rather than strengthens their public messages." In this respect, the need for clarity and thorough information about the pandemic worldwide has promoted the birth of several multilingual projects aimed at providing as many countries as possible with a standard vocabulary that can contribute to favouring full and correct understanding of the COVID-19 pandemic. ${ }^{1}$ In this respect, one of the most important endeavours is the Oxford Languages Multilingual COVID-19 Project, which offers key COVID-19 terminology translations from English into 19 different languages, together natively spoken by over $40 \%$ of the worldwide population. As the authors of the project state, "the translations are freely available in order to support local efforts to provide critical health advice to various communities around the world" (https://languages.oup.com/covid-19-language-resources/). The authors also highlight that "the translations may also be of interest to those wishing to study how different languages are evolving as a result of the pandemic" (ibid.). The corpus used for the project includes COVID-19-related scientific terms

\footnotetext{
${ }^{1}$ This issue is strictly connected with that of the importance of multilingual and intercultural competence in a globalized world (see Alaoui 2017).
} 
belonging to the fields of medicine, epidemiology, and biology. The corpus also includes terms and expressions concerning government and actions aimed at limiting the spread of the virus and its social and economic consequences. Another important project is that of Harvard Medical School, Harvard University, and Division of Pulmonary and Critical Care Medicine, Brigham and Women's Hospital, Boston, Massachusetts, aimed at diffusing COVID19-related information to those segments of population, i.e. school-aged children and teenagers, that have been left isolated from accurate information about the pandemic. ${ }^{2}$ Moreover, the material has been translated into Spanish, given that $20 \%$ of the U.S. population is represented by non-native Englishspeaking individuals, the majority being Hispanic/Latinx who do not speak English or have LEP. The material has also been translated into French as this was the second language with the highest number of page views after English in 2020 .

\section{COVID-19 information material and linguistic minorities}

The question of minority groups' access to COVID-19-related information has been largely debated over the last year and much attention has been paid to the increasing evidence that these groups are more prone to be affected by COVID-19 (Do 2020; Germain and Yong 2020; Khan et al. 2020; Platt and Warwick 2020). Do (2020) offers a list of the main factors that expose minority groups to a higher risk of being infected by SARS-CoV-2. They include discrimination; healthcare access and utilization; occupation; educational, income and wealth gaps; and housing. When dealing with the issue of healthcare access and utilization, Do (2020), who makes specific reference to American society, provides an in-depth analysis that encompasses the several aspects limiting access to the healthcare system for minority groups, ranging from cultural differences between patients and providers to distrust towards the government and healthcare systems considered responsible for inequities in treatment, from the opportunity to take some time off from work to communication and language barriers. With reference to the latter aspect, this paper will focus on the importance of translation and multilingual information material availability as considered fundamental in helping minority language groups weather the setbacks that hinder their access to the healthcare system and get adequate understanding of the necessary measures to prevent them from getting infected.

Translation is the basic means of intercultural communication and the role it plays in a context of global health crisis is fundamental. Zhang and $\mathrm{Wu}$ (2020: 518-519) highlight how important translation was, especially in the

\footnotetext{
${ }^{2}$ Shahid et al. (2020) carried out a research on the question of incorrect and misleading information about the COVID-19 pandemic, with particular reference to misinformation from social media platforms.
} 
early stage of the ongoing pandemic, in facilitating international donations and the procurement of medical materials between Wuhan and the world. Piller, Zhang and $\mathrm{Li}$ (2020: 503) say that "public health communication is characterized by the large-scale exclusion of linguistic minorities from timely high-quality information." Khan et al. (2020) carried out a research to investigate presence and readability of translated online information available to the British public during the COVID-19 pandemic, with particular attention to BAME (Black and Minorities Ethnicities). They found out that the readability of information was below national standards and that there was lack of accompanying graphic-based material.

The aim of the present study was to carry out an investigation of COVID-19-related information material as displayed on the websites of governmental and non-governmental sources in Italy, a country with an increasing number of foreigners, the majority coming from non-European countries. In this respect, Italy is the fourth European country for number of migrants, following Germany, Spain and France (Eurostat, Statistics Explained 2021, https://ec.europa.eu/eurostat/statisticsexplained/). It has a growing foreign population amounting to more than 5.1 million registered inhabitants. These include Romanians (the most consistently represented foreign population in Italy), Albanians, Moroccans, Ukrainians, Chinese, Filipinos, Bangladeshi, Pakistani, and Indians (Istat, Istituto Nazionale di Statistica, https://www.istat.it/it).

\section{Corpus and Methodology}

The investigation took into account the websites of Ministero della Salute (Ministry of Health), Istituto Superiore di Sanità (Italian National Institute of Health), and Il Grande Colibrì volunteer association. The analysis evaluated availability of multilingual material concerning COVID-19, types of content, graphic-based material as considered a supporting factor in text readability, and clarity of information. As for the latter feature, in particular, the linguistic analysis was carried out only for the English language that, given its status as an international medium of communication, was included in all the multilingual material found. Moreover, for most linguistic minorities living in Italy, English represents either the most studied foreign language (e.g. in Romania) or one of the official languages of their country (e.g. in Pakistan). The analysis of the material in the English version did not consider readability formulas (e.g SMOG, Gunning Fog Index, Flesch Kincaid) but a user-centred approach that took into account the target users' actual needs. In the specific case of this study, the users' needs lie in the availability of a plain language, that is a language that can be accessible to as many people as possible, also given that not all foreigners living in Italy have high English proficiency and literacy levels. In this respect, Jarrett and Redish (2019) report that the concept 
of plain language has shifted from writing short sentences and words to focusing on the people who read what is written, i.e., on people's knowledge. For this reason, the two scholars condemn the use of readability formulas to assess the level of intelligibility of a text. They maintain that readability formulas are neither reliable nor valid as they basically consider word and sentence length as indicators of lexical and syntactical complexity, and this may be misleading. They say: "If a formula only counts word length, it does not consider whether your readers are likely to know what the words mean" (Jarrett and Redish 2019). If syntactical complexity and sentence length may affect comprehension, the lexical aspect is a major concern. A short word is not automatically easy to understand just because it is short. The comprehension of its meaning basically depends on whether that word is part of one's L1 repertoire and, to a greater extent, of one's L2 competence - when the word belongs to a foreign language. The issue becomes crucial when dealing with health communication - and even more so with global crises like the current pandemic - for which language access must be deemed a priority to disseminate urgent information to all demographic communities. To assess the readability of the English material the Plain Language Guidelines (2011) was used, a work developed by the Plain Language Action and Information Network (PLAIN), a community of US federal employees who claim transparency and clarity of information for communication from the Government. The guidelines in question take inspiration from the Plain Writing Act of 2010 where plain language (also referred to as plain writing or plain English) is defined as "writing that is clear, concise, well-organized, and follows other best practices appropriate to the subject or field and intended audience" (https://www.govinfo.gov/content/pkg/PLAW-111publ274/pdf/PLAW111publ274.pdf).

Based on this tenet, the guidelines define the linguistic characteristics that any material must comply with to be considered as written in "plain language". These are the following:

- reader-centred organization

- use of "you" and other pronouns

- active voice

- common words

- easy-to-follow design features (lists, headers, tables)

The investigation mainly focused on information concerning the COVID-19 restrictive measures set up by the Italian government for the national territory and representing the most important actual information needed by minority groups living in Italy. As a matter of fact, it was assumed that foreign people looking for general information about COVID-19 could 
easily obtain it from a multitude of online sources and in their native language. By contrast, thorough information concerning rules, decrees, and ordinances in force in Italy could be retrieved only by consulting Italian official websites, and basically in two ways, namely either by means of possible material translated into their native language or into the language of international communication - therefore English. The latter was expected to be plain English so as to guarantee information access to as many foreign people as possible.

\section{Results}

Comparison of the aforementioned websites revealed significant differences as for several factors. First and foremost, remarkable dissimilarities regarding the multilingual material displayed can be found, in that not all the main native languages spoken by foreigners living in Italy are represented in the two websites of Ministero della Salute and Istituto Superiore di Sanità. In Ministero della Salute, the Italian material has been translated only into English, and the Italian sections including material related to COVID-19 information have not all been translated into English. More precisely, the material in the Italian language is divided into 13 sections, but only 4 of them display their corresponding sections in the English version. They are "COVID-19, situation in Italy", "COVID-19, situation in the world", "COVID-19 Vaccine Plan", and "COVID-19 Travellers". The 9 remaining sections, which include, among the others, "Technical Scientific Committee" and "Donations" have not been translated, probably because containing information not specifically pertaining to the Italian territory and therefore barely motivating for foreign people. Moreover, the website offers a FAQ section in English with over 100 COVID-19-related questions, and a daily report with a dashboard map, a daily data sheet an epidemiologicalsurveillance infographic (the latter being a link to material created by Istituto Superiore di Sanità).

Istituto Superiore di Sanità provides a "Special on Covid-19" section in Italian and English but not all the information available in Italian has been translated into English, e.g. rules for isolation, quarantine, and close contacts. The website also provides a FAQ section including statistical and epidemiological data in English, and a multilingual practical guide specifically designed for caregivers of elderly people. The material offers information about what COVID-19 is, the symptoms of the disease, how the virus is transmitted, how to take care of an elderly person, and in-depth practical advice on domestic hygiene with particular attention to hand washing procedures. The guide has been translated into 7 languages, namely English, Spanish, French, Portuguese, Romanian, Polish and Russian (Chinese and Filipino are not included). However, the accessibility to the multilingual 
material is not immediate as the information is not provided on the homepage of the website and quite a long procedure is required to find it.

By contrast, easy accessibility to multilingual material is that provided by such volunteer associations as Il Grande Colibrì, which offers instructions in more than 50 different languages, including non-European idioms like Somali, Turkish, Urdu, Pashtu, and Wolof. The material concerns behavioural protocols to be followed in Italy and is continuously updated to allow users to keep up with rules and restrictions that often change according to the colours (red, orange, yellow, and white) of regions and autonomous provinces, set up depending on risk levels. The texts are accompanied by abundant graphic material, characterized by well-defined illustrating images.

As for the type of English used in the information material provided by the websites, some questions included in the FAQ section of Ministero della Salute were investigated, namely "What containment measures are planned in Italy" and "What is recommended to Travellers?", two issues that foreign groups living in Italy must know very well.

\section{"What containment measures are planned in Italy"}

On April 26, 2021, Decree-law n. 52 of April 22, 2021 came into force, introducing urgent measures for gradual resumption of economic and social activities, in compliance with the need to contain the spread of the COVID-19 epidemic. The text outlines the timetable for the gradual elimination of the necessary restrictions to limit contagion from the SARS-CoV-2 virus, in the light of scientific data on the epidemic and the progress of the vaccination campaign.

\section{Green certifications}

The decree-law introduces, throughout the country, the so-called 'Covid-19 green certifications', proved that vaccination against SARS-CoV-2 has been carried out, or that the patient has recovered from the infection, or has undergone a rapid molecular or antigenic test with a negative result.

\section{Classification of Regions and Autonomous Provinces}

Regions and Autonomous Provinces are classified into four areas, according to the data and indications of the Control Room/Cabina di Regia (DM April 30, 2020). Each area corresponds to different epidemiological risk scenarios and levels, according to the document "Prevention and response to COVID-19; evolution of the strategy and planning in the transition phase for the autumn-winter period", and the restrictions of the last decree - Dpcm: 
- the most restrictive measures are concentrated in the red area, referring to the Regions and Autonomous Provinces with a scenario of maximum severity and a high level of risk;

- in the orange area, including the Regions and Autonomous Provinces with a high severity scenario and a high-risk level, there are slightly less restrictive measures;

- in the yellow area there are general restrictive measures;

- in the white area there are no restrictions.

The classification of the Regions into areas is established by Ordinance of the Minister of Health, after consulting the Presidents of the Regions concerned.

\section{Movements}

Among the new features of the Decree-Law of April 22, as of April 26 travel between different regions in the white and yellow areas is permitted. In addition, people with a "green certification" are also allowed to travel between Regions and Autonomous Provinces in the orange or red areas.

From April 26 to June 15, 2021, in the yellow area, four people are allowed to move to a single inhabited private home once a day, from 5 am to $10 \mathrm{pm}$, in addition to those already living in the home of destination. Persons moving may bring with them minors over whom they exercise parental responsibility and disabled or dependent persons living with them.

The same movements, with the same hourly limits and number of persons, is allowed in the orange area, within the same municipality. Travel to other private dwellings in the red area is not permitted.

(Ministero della Salute, http://www.salute.gov.it/portale/nuovocoronavirus/dettaglioFaqNuovoCoron avirus.jsp?lingua $=$ english $\&$ id $=230 \# 11$ )

\section{What is recommended to travellers?}

Travel rules vary, depending on the country of origin or destination and the reasons for travelling. With the Ordinance 29 Aprile, 2021 the Minister of Health updated the cross-border measures with reference to entries from India, Bangladesh and Sri Lanka and extended the existing measures for entries from List C, List D and List E countries until 15 May 2021.

\section{India, Bangladesh, and Sri Lanka}

It is forbidden to enter Italy for people coming from or who have stayed in the previous fourteen days in India, Bangladesh, and Sri Lanka, with the exception of Italian citizens who have been registered in Italy since before April 29, 2021 and who do not show COVID-19 symptoms, and of the subjects 
referred to in Article 51, paragraph 7, letter n) of the Ministerial Decree of March 2, 2021.

The above categories may be authorized entry exclusively in compliance with a specific protocol. The Ordinance of April 2, 2021 provided for some reclassifications on the lists of countries in Annex 20 of the Dpcm of March 2, 2021.

(Ministero della Salute, http://www.salute.gov.it/portale/nuovocoronavirus/dettaglioFaqNuovoCoron avirus.jsp?lingua $=$ english \&id $=230 \# 11$ )

As can be seen, both texts above treat a topic that a foreign audience needs to know, that is restrictive measure-related changes in Italy depending on the risk level of the various regions. However, the discourse reveals a technical and objective description of the topic dealt with; personal pronouns are never used, the use of passivation is quite pervasive, and the vocabulary includes words and expressions that may not be of immediate comprehension as they do not belong to the basic English lexicon (e.g. features, dwellings, undergone, forbidden, in compliance with, etc.). Moreover, the discourse reveals a mostly complex syntactic structure, with long and complex sentences.

The same linguistic features can also be found when dealing with the general recommendations that all citizens must respect:

\section{What is recommended to all Italian citizens?}

- People showing symptoms, even mild ones, such as fever, sore throat, cough, rhinorrhoea/nasal congestion, difficulty in breathing, muscular pains, anosmia/ageusia/dysgeusia, diarrhoea, asthenia, should stay at home and immediately contact their general practitioner or paediatrician:

- Total ban on mobility from home and social contacts for persons under quarantine;

- Gatherings of people in public places or places open to the public is forbidden;

- Frequent hand washing (with soap and water or an alcoholic solution), proper respiratory hygiene (coughing and sneezing directly into a tissue or into the crook of the elbow) is recommended;

- It is mandatory to respect the recommended safety distance of at least 1 metre between one person and another;

- It is mandatory to always wear a mask, throughout the national territory;

- The use of a mask is mandatory;

- $[\ldots]$ 
- Masks are not mandatory in the following cases:

- people taking part in sporting activities;

- children under six years of age;

- people with pathologies or disabilities that are incompatible with the use of a mask, and those who have the same incompatibility when interacting with them;

- The use of a face mask is also strongly recommended in private homes, if there are non-residents visiting

It is also advised that all citizens respect simple preventive recommendations.

(Ministero della Salute, http://www.salute.gov.it/portale/nuovocoronavirus/dettaglioFaqNuovoCoron avirus.jsp?lingua=english\&id=230\#11).

What can immediately be noticed in the extract above is the abundant use of medical terms to describe the possible symptoms caused by COVID19. Such terms as rhinorrhoea, anosmia, dysgeusia belong to specialized lexicon and may also be obscure for people who do have a good command of English but are not familiar with medical terminology. Moreover, discourse is depersonalised due to the presence of indirect forms (e.g. it is mandatory). However, when dealing with the symptoms of COVID-19, specialised terms are displayed in brackets and their explanation is provided:

Symptoms of COVID-19 may vary, according to the severity of the disease, from no symptoms (being asymptomatic) to fever, cough, sore throat, weakness, fatigue, and muscle pain. More severe cases may involve pneumonia, acute respiratory distress syndrome and other complications, all of which can be life-threatening.

Sudden loss of sense of smell (anosmia) or diminished sense of smell (hyposmia), loss of taste (ageusia) or taste alteration (dysgeusia) have been recognised as symptoms of COVID-19.

Other less specific symptoms may include headache, chills, myalgia, asthenia, vomiting and/or diarrhoea.

(Ministero della Salute, http://www.salute.gov.it/portale/nuovocoronavirus/dettaglioFaqNuovoCoron avirus.jsp?lingua $=$ english\&id $=230 \# 2$ )

As can be seen, most medical terms are preceded by the definition of their meaning but the effort does not encompass all terms, as shown in the last sentence, where myalgia and asthenia are not accompanied by any explanation. As for the description of the symptoms included in the website of the Istituto Superiore di Sanità, these are displayed in the form of a list contained in a guide for caregivers of elderly people: 
What are the symptoms of coronavirus? The disease can affect people differently; sometimes an infected person does not show any symptoms. At other times, these symptoms may resemble those of a cold or a flu:

- fever

- muscle pain

- nasal congestion

- sore throat

- cough

- difficult breathing (dyspnea)

- eye inflammation

- diarrhea

- severe headaches

- loss of smell

- loss of taste

(Istituto Superiore di Sanità, https://www.iss.it/documents/20126/0/20200423_ISS_Opuscolo+Covid19_B adanti_EN.pdf/69e7a4ed-13f6-a2ce-6943-

$54 \mathrm{f} 2 \mathrm{ddb} 6 \mathrm{ede} 3 \mathrm{t}=1587655016271)$.

The list is followed by an explanation, in a very descriptive form, of how the virus is transmitted, and a semi-colloquial tone is kept, with use of possessives and personal pronouns:

How is the coronavirus transmitted? The virus is transmitted through small droplets of saliva, which we all emit through the mouth and nose when speaking, sneezing, or coughing. If the droplets containing the virus come into contact with our mouth, nose or eyes, infection may occur (that's why it is important to maintain a safety distance of at least one metre). Droplets from an infected person can also stick to their hands, or to nearby objects. Therefore, even objects that have been touched by an infected person may contain the virus (and that's why it is important to wash your hands frequently).

(Istituto Superiore di Sanità, https://www.iss.it/documents/20126/0/20200423_ISS_Opuscolo+Covid19_B adanti_EN.pdf/69e7a4ed-13f6-a2ce-6943-54f2ddb6ede3?t=1587655016271)

COVID-19-related information containing all aspects referred to above with regard to plain English as described in the Plain Language Guidelines (2011) can be found on the website of Il Grande Colibrì volunteer association, where information displays a clearly reader-centred organization of discourse and easy-to-follow linguistic features

Coronavirus In Italy: What To Do

ATTENTION! THERE ARE DIFFERENT RULES IN THE DIFFERENT ITALIAN REGIONS! CHECK WELL IN THE PAGES BELOW! 
(Il Grande Colibrì, https://www.ilgrandecolibri.com/wpcontent/uploads/2020/02/20201110-inglese.pdf)

The information, always written in capital letters, is organized in small chunks of text accompanied by illustrating images, and common words are used. The first information provided is introductory and explains what COVID-19 is:

THE NEW CORONAVIRUS IS A VIRUS THAT CAUSES COVID-19 DISEASE. (ibid.)

The following information immediately focuses on the seriousness of the disease and its consequences:

\section{THIS DISEASE CAUSES MAJOR HEALTH PROBLEMS. SOMETIMES} IT ALSO LEADS TO DEATH. (ibid.)

The first two sentences above show a linear thematic progression of information: the rheme in sentence 1 becomes the theme in sentence 2., and this gives information advancement its most basic and straightforward form. After proceeding with information about the main symptoms of the disease, and using short sentences, simple words, and personal pronouns and possessives, as in the sentences:

IF YOU HAVE FEVER, COUGH, OR HAVE TROUBLE BREATHING DON'T LEAVE THE HOUSE, CALL YOUR DOCTOR AND DO WHAT HE/SHE SAYS (ibid.)

and

EVEN IF YOU FEEL GOOD YOU SHOULD LEAVE THE HOUSE ONLY TO GO TO SCHOOL, WORK OR TO GO SHOPPING (ibid.).

The text develops in a thematic progression with a constant theme:

SOME INFECTED PEOPLE ARE NOT AWARE THAT THEY GOT THE VIRUS BECAUSE THEY MAY NOT HAVE HEALTH PROBLEMS. THESE INFECTED PEOPLE SPREAD THE VIRUS TO OTHERS WHEN THEY COUGH, TALK, OR BREATHE.

WHEN AN INFECTED PERSON TOUCHES AN OBJECT, THE CORONAVIRUS STICKS TO THE OBJECT. (ibid.)

The reiteration of the same theme occurs by means of lexical repetition (infected people/person, object); this also serves the important function of reinforcing the communicative effectiveness of the message to be conveyed, as also shown in the following information:

WASH YOUR HANDS VERY OFTEN DURING THE DAY. DON'T TOUCH YOUR FACE BEFORE WASHING YOUR HANDS. (ibid.)

As far as the material provided by the website is concerned, the introductory part to the characteristics of SARS-CoV-2 is followed by 
explanation of the different rules in force in Italy depending on risk levels (classified into "very high risk", corresponding to red zones, "high risk", orange zones, and "medium risk", yellow zones). Thus, with reference to yellow zones, the following information can be found:

FROM 10 P.M. TO 5 A.M., YOU CAN LEAVE THE HOUSE ONLY TO GO TO WORK, FOR HEALTH PROBLEMS OR EMERGENCIES. YOU MUST ALWAYS WEAR THE MASK.

FROM 5 A.M. TO 10 P.M., YOU CAN LEAVE THE HOUSE, BUT YOU MUST ALWAYS WEAR THE MASK.

YOU MUST ALWAYS BE AT LEAST ONE METER APART FROM NON-COHABITATING PEOPLE.

YOU CAN PRACTICE SPORT ALONE IN THE STREETS OR IN THE PARKS, BUT YOU MUST BE AT LEAST TWO METERS APART FROM OTHER PEOPLE. (ibid.)

The needs of the target audience seem to have been fulfilled as for discourse features, namely use of common words and of the personal pronoun "you", short sentences, active forms, as well as the repeated use of some words, including the deontic modal "must", which also serves a persuasive function.

\section{Conclusion}

Language, translation, and multilingual information play a fundamental role in a global health crisis context. Compliance with prevention rules, protocols and instructions is necessary to limit the spread of an ongoing infection. In this respect, efforts to defeat language barriers represent a crucial factor that contributes to overcoming distrust in the healthcare system. In Italy, besides English as the language of international exchange, governmental and non-governmental institutions should provide COVID-19 material in the languages spoken by the main (if not all) minority groups living in the country. In this respect, high-quality crowdsourcing translation projects could be of help, especially when great amounts of material need to be translated in a short time. If English is chosen as the only available foreign language, information should be conveyed in plain language to allow easy access to key knowledge. Plain language does not mean simplified language, nor does it mean lack of complex information. It means a language where the focus is on the message rather than on the language itself. 


\section{References:}

1. Alaoui, S. M. (2017). Promoting Multilingual Communicative Competence for the Labor Market. European Scientific Journal, ESJ, 13 (7), (pp. 201-214).

2. Cappuzzo B. (2020) Anglicisms and Italian Equivalents in the Era of Covid-19: A Corpus-Based Study of Lockdown. In F. Daniele (ed.), The language of Pandemics, European Scientific Journal, ESJ, Special Edition, 16 (38), (pp. 7-26).

3. Deang R. K. and D. Salazar (2021), Major Health Crisis and the OED: Language Evolution and Challenges in Health Communication, https://public.oed.com/blog/major-health-crises-and-the-oed/ (last accessed May 2021).

4. Do W.W.C. (2021) Health Equity Considerations and Racial and Ethnic Minority Groups, https://www.cdc.gov (last accessed May 2021).

5. Eurostat, Statistics Explained 2021, https://ec.europa.eu/eurostat/statisticsexplained/ (last accessed May 2021).

6. Germain S. and A. Yong (2020), COVID-19 Highlighting Inequalities in Access to Healthcare in England: A Case Study of Ethnic Minority and Migrant Women, Feminist Legal Studies 28, (pp. 301-310).

7. Istat, Istituto Nazionale di Statistica, https://www.istat.it/it (last accessed May 2021).

8. Jarrett C. and J. Redish (2019), Readibility Formulas: 7 Reasons to Avoid Them and What to Do Instead, https://www.uxmatters.com/mt/archives/2019/07/readabilityformulas-7-reasons-to-avoid-them-and-what-to-do-instead.php (last accessed May 2021).

9. Khan S., Asif A. and A.E. Jaffery (2020), Language in a Time of COVID-19: Literacy Bias Ethnic Minorities Face During COVID-19 from Online Information in the UK, Journal of Racial and Ethnic Health Disparities, https://doi.org/10.1007/s40615-020-00883 (last accessed May 2021).

10. Il Grande Colibrì, https://www.ilgrandecolibri.com/ (last accessed May 2021).

11. Istituto Superiore di Sanità, https://www.iss.it/ (last accessed May 2021).

12. Ministero della Salute, https://www.salute.gov.it/portale/home.html (last accessed May 2021).

13. Oxford English Dictionary Online (OED), Oxford: O.U.P., http://www.oed.com (last accessed May 2021). 
14. Oxford Languages Multilingual COVID-19 Project, https://languages.oup.com/covid-19-language-resources/ (last accessed May 2021).

15. Piller I., Zhang J. and J. Li (2020), Linguistic Diversity in a Time of Crisis: Language Challenges of the COOVID-19 Pandemic, Multilingua 39 (5), (pp. 503-515).

16. Plain Language Guidelines (2011), https://www.plainlanguage.gov/media/FederalPLGuidelines.pdf (last accessed May 2021).

17. Plain Writing Act of 2010, https://www.govinfo.gov/content/pkg/PLAW-

18. 11 publ274/pdf/PLAW-111publ274.pdf (last accessed May 2021).

19. Platt L. and R. Warwick (2020), Are Some Ethnic Groups More Vulnerable to COVID-19 Than Others?, Inequality. London: The Institute for Fiscal Studies, (pp. 1-26).

20. Shahid, S., Habibullah Mangrio, R., Abbas, M., Ali, Z. and M. Ahmed (2020). Evaluation of Knowledge and Belief on False Reports and Misinformation from Social Media in COVID-19 Pandemic: A Web Based Cross-Sectional Survey in Karachi, Pakistan. European Scientific Journal, ESJ, 16 (36), (pp. 8-17).

21. Treccani, www.treccani.it (last accessed May 2021).

22. Zhang J, and Y. Wu (2020), Providing Multilingual Logistics Communication in Covid-19 Disaster Relief, Multilingua 39 (5), (pp. 517-528). 